\title{
Tratamento da recidiva da leucemia mielóide crônica após transplante de medula óssea alogênico utilizando mesilato de imatinibe: Relato de três casos Treatment of chronic myelogenous leukemia relapse after allogeneic bone marrow transplantation with imatinib mesylate: Report of three cases
}

Ronald Pallotta ${ }^{1}$

Denize F. Lima²

Flávia Cal ${ }^{3}$

Maiana Almeida ${ }^{4}$

Mônica Conchon 5

\begin{abstract}
O mesilato de imatinibe (MI), inibidor seletivo da tirosinoquinase envolvido na patogênese da leucemia mielóide crônica (LMC), tem se constituído como terapia farmacológica de primeira linha para o tratamento desta doença. A infusão de linfócitos do doador (DLI) tem sido considerada como tratamento padrão para recidiva da LMC após transplante de medula óssea (TMO) alogênico, apesar de estar freqüentemente associado à ocorrência de doença do enxerto contra hospedeiro e mielossupressão. Por apresentar resultados satisfatórios e boa tolerabilidade no tratamento da LMC, os autores empregaram o mesilato de imatinib como terapêutica alternativa à DLI em pacientes que sofreram recidiva após o TMO. Obtiveram sucesso em dois casos, sendo que em um houve retorno comprovado do quimerismo do doador. No terceiro caso houve progressão da doença e o paciente foi encaminhado para segundo TMO. Desta forma, devido ao caráter recente do tema, este estudo descritivo sugere que esta opção terapêutica possa ser estudada como alternativa na recaída pós-TMO. Rev. bras. hematol. hemoter. 2006;28(2):157-160.
\end{abstract}

Palavras-chave: Leucemia mielóide crônica, tratamento, recidiva, transplante de medula óssea, mesilato imatinibe.

\section{Introdução}

A leucemia mielóide crônica (LMC) representa cerca de $15 \%$ do total das leucemias, acometendo em torno de um a dois casos para cada 100 mil indivíduos anualmente. ${ }^{1,2}$ Apresenta um pico de incidência entre 45 e 55 anos de idade, podendo acometer crianças e, em $10 \%$ a $15 \%$ dos casos, pacientes a partir de 60 anos. $^{3}$ É uma doença mieloproliferativa crônica desencadeada na sua grande maioria a partir de uma translocação recíproca entre os braços longos dos cromossomos 9 e 22 (t 9;22), denominado cromossomo Philadelphia (Ph1). ${ }^{1,2}$ Essa translocação resulta na fusão do gene BCR (breakpoint cluster region) no cromossomo 22q11, com o gene ABL (Ableson leukemia vírus), localizado no cromos- somo 9q34., ${ }^{1,2,3,4} \mathrm{O}$ oncogene híbrido bcr/abl resultante desta aposição promoverá a síntese de uma proteína com atividade tirosinoquinase, responsável por estímulos à proliferação e ao bloqueio na apoptose da célula progenitora hematopoética. ${ }^{1,3,5}$

O arsenal terapêutico da LMC está compreendido na escolha racional entre a terapia celular, através do transplante de medula óssea (TMO), e o tratamento medicamentoso. Este último vem sofrendo notáveis transformações e sendo motivo de muitos estudos, principalmente após o surgimento, há sete anos, do mesilato de imatinibe (MI). ${ }^{5} \mathrm{O}$ MI age como um inibidor específico da proteína com atividade tirosinoquinase, produzida pelo gene bcl/abl, impedindo a fosforilação de substratos envolvidos na regulação do ciclo

\footnotetext{
${ }^{1}$ Professor adjunto da Escola Bahiana de Medicina e Saúde Pública e Coordenador do Centro de Transplante de Medula Óssea da Bahia. ${ }_{3}^{2}$ Estágiária de Ciências Biológicas da Escola Bahiana de Medicina e Saúde Pública.

${ }^{3}$ Médica estagiária da Escola Bahiana de Medicina e Saúde Pública.

${ }_{5}^{4}$ Acadêmica da Escola Bahiana de Medicina e Saúde Pública.

${ }^{5}$ Médica hematologista responsável pelo Serviço de Citogenética do Hospital das Clínicas da FMUSP.
}

Correspondência: Ronald Pallotta

Av. Oceânica 3.529 - Ondina

40170010 - Salvador-BA

E-mail: tmobahia@yahoo.com.br 
celular. ${ }^{5,6,7}$ Dessa forma, tem sido considerada a droga de primeira linha para os pacientes não candidatos ao TMO, ${ }^{7}$ assim como para aqueles recém-diagnosticados com risco elevado para terapia celular. ${ }^{5,8,9,10}$

O TMO ainda é considerado, por muitos autores, como único tratamento capaz de promover cura para LMC. ${ }^{9-14} \mathrm{~A}$ chance de cura oscila em torno de $65 \%$, 3,7 podendo atingir uma taxa de sobrevida de $70 \%$ em dez anos. ${ }^{3}$ Entretanto, essas taxas podem variar a depender da idade, compatibilidade com o doador, combinação de sexo, tempo entre o diagnóstico e o transplante, e a fase da doença em que se encontra. ${ }^{15}$ Apesar de seu poder de cura, somente $15 \%-30 \%$ dos pacientes serão candidatos a este procedimento, tendo como principais limitantes a idade e a indisponibilidade de doador compatível. ${ }^{2,3,7,8}$ Além disso, as taxas de recidiva após o TMO variam de 5\%-30\% na fase crônica, podendo chegar a $60 \%$ nas fases acelerada e blástica. ${ }^{15}$

Os pacientes com recidiva após o TMO vêm sendo tratados, nos últimos anos, com o uso de interferon alfa, quimioterapia ou um segundo TMO. No entanto, a infusão de linfócitos do doador (DLI), através da reação enxerto contra leucemia, tem se constituído, até então, o tratamento de primeira linha. ${ }^{13-17}$ Uma resposta molecular completa tem sido alcançada em até $60 \%-70 \%$ dos pacientes em fase crônica com tal recurso terapêutico..$^{15}$ Apesar de sua alta capacidade de resposta molecular, o uso da DLI traz consigo as complicações da mielossupressão e a considerável morbi-mortalidade da doença enxerto contra hospedeiro (DECH), presente em mais de $60 \%$ desta população, o que tem limitado sua aplicação em um número considerável de pacientes. ${ }^{13-16}$ Devido aos bons resultados apresentados com o uso do MI, tem-se cogitado a hipótese de seu uso como tratamento alternativo a DLI em pacientes com recidiva. ${ }^{13-16,18,-22}$ Dessa forma, esse estudo descreve três casos referentes a pacientes portadores de LMC que obtiveram a recidiva da doença após o TMO e seguem o tratamento com o mesilato de imatinibe.

\section{Relato dos Casos}

De agosto de 2000 a agosto de 2005, o CTMO da Bahia acompanhou vinte pacientes portadores de LMC que foram transplantados. Destes pacientes, três (15\%) apresentaram recidiva e foram tratados com mesilato de imatinibe.

\section{Caso 1}

Paciente do sexo masculino, 33 anos, negro, previamente saudável, teve diagnóstico de LMC cromossomo Ph1 positivo em maio de 1997. Inicialmente em fase crônica, vinha fazendo uso de hidroxiuréia sem resposta ao tratamento. Em 2000 foi submetido a TMO alogênico com um score de três fatores de risco para o procedimento pelo European Group for Blood and Bone Marrow Transplantation (EBMTR). O regime de condicionamento utilizado foi com bussulfano (16 mg/kg) e ciclofosfamida (120 mg/kg). Recebeu em 23/03/ 2001, 2,8 x $10^{8}$ células nucleadas totais por kg da sua irmã HLA totalmente compatível. Foi realizada profilaxia da DECH com metotrexato $15 \mathrm{mg} / \mathrm{m}^{2}$ no dia +1 e $10 \mathrm{mg} / \mathrm{m}^{2}$ nos dias
$+3,+6$ e +11 aplicados por via endovenosa associado ao uso de ciclosporina. Apresentou como complicação pós-transplante DECH crônica de novo em cavidade oral, olho, boca, couro cabeludo e fígado, fazendo uso de ciclosporina e predinisona para controle da mesma. O paciente obteve o controle hematológico e citogenético da doença de base até julho de 2004, quando apresentou recidiva clínica e hematológica. Nesta época, a citogenética demonstrava vinte das vinte metáfases analisadas com 46XY, com presença do cromossomo Ph1 em 100\%, confirmando a recidiva em células do receptor. Tendo em vista a presença da DECH crônica e a indisponibilidade imediata da doadora, iniciou, então, o uso de MI 400 mg.

Tabela 1

Evolução laboratorial da leucometria do caso 1

\begin{tabular}{cccccc}
\hline & Pré-MI & $3^{\circ}$ mês & $6^{\circ}$ mês & $9^{\circ}$ mês & $12^{\circ}$ mês \\
$\begin{array}{c}\text { Leucócitos } \\
\mathrm{mm}^{3}\end{array}$ & 66100 & 5300 & 3800 & 4000 & 4200 \\
\hline
\end{tabular}

Paciente apresentou boa aceitação e evoluiu com controle hematológico da doença (Tabela 1). Na avaliação de 6 meses após a introdução do MI, o cariótipo demonstrou 100\% das metáfases $46 \mathrm{XX}$, confirmando a resposta completa citogenética e o restabelecimento do quimerismo da doadora, que permaneceu na avaliação do primeiro ano (Gráfico1).

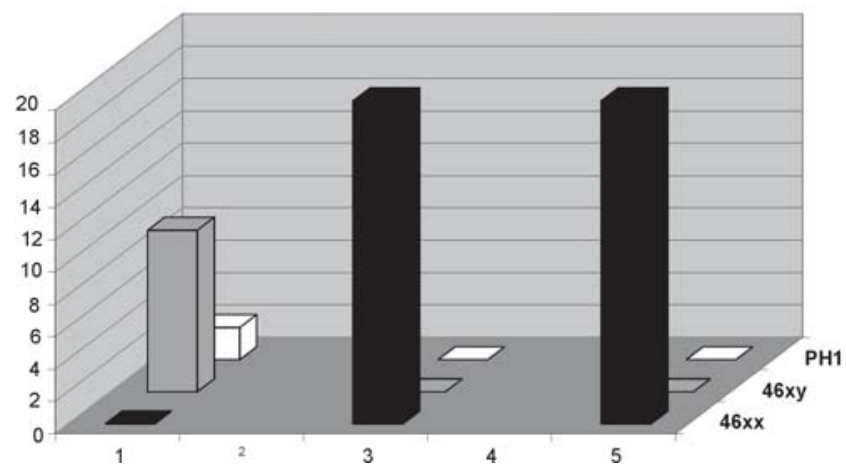

Gráfico 1. Evolução citogenética do caso 1

\section{Caso 2}

Paciente do sexo masculino, 31 anos, branco, procurou assistência médica, em setembro de 1996, com quadro de dor em hipocôndrio esquerdo, quando obteve o diagnóstico de LMC cromossomo Philadelphia positivo em fase crônica. Fez uso de hidroxiuréia e interferon sem sucesso no controle da doença. Em novembro de 1999, foi submetido a TMO alogênico com um score de dois fatores de risco para o procedimento pelo EBMTR. O regime de condicionamento utilizado foi com bussulfano (16 mg/kg) e ciclofosfamida (120 mg/kg) e em 12/11/2000 recebeu 2,04 x $10^{8}$ células nucleadas totais por kg de seu irmão HLA totalmente compatível. A profilaxia da DECH foi com metotrexato $15 \mathrm{mg} / \mathrm{m}^{2}$ no dia +1 e $10 \mathrm{mg} / \mathrm{m}^{2}$ nos dias $+3,+6$ e +11 aplicados por via endovenosa associado ao 
uso de ciclosporina. Não apresentou doença do enxerto contra hospedeiro evoluindo bem, sem intercorrências, até maio de 2002 (um ano e cinco meses pós-TMO), quando apresentou recaída hematológica da doença. A avaliação citogenética demonstrou a presença do cromossomo Philadephia em dez das vinte metáfases analisadas. Desta forma, foi reiniciado o tratamento com hidroxiuréia e, como não havia sinais de DECH, foi realizado DLI sendo a primeira infusão em maio de 2002 com 1 x $10^{7}$ células CD3/kg, a segunda infusão em setembro de 2002 com $5 \times 10^{7}$ células CD3/kg e a terceira infusão em novembro de 2002 com 0,5 x $10^{8}$ CD3 $/ \mathrm{kg}$.

Infelizmente o paciente não obteve resposta ao tratamento oferecido mantendo leucocitose no sangue periférico e progressão do perfil citogenético para 100\% de metáfases com presença Ph1+. Desta forma, foi iniciada então a terapia com MI $400 \mathrm{mg} / \mathrm{d}$.

Paciente evoluiu com controle hematológico da doença (Tabela 2) e citogenético (Gráfico 2).

Tabela 2

Evolução laboratorial da leucometria do caso 2

\begin{tabular}{|c|c|c|c|c|c|}
\hline & Pré-MI & $3^{\circ}$ mês & $6^{\circ}$ mês & $9^{\circ}$ mês & $12^{\circ}$ mês \\
\hline $\begin{array}{c}\text { Leucócitos' }^{\prime} \mathrm{mm}^{3}\end{array}$ & 14230 & 5000 & 4900 & 4900 & 4900 \\
\hline
\end{tabular}

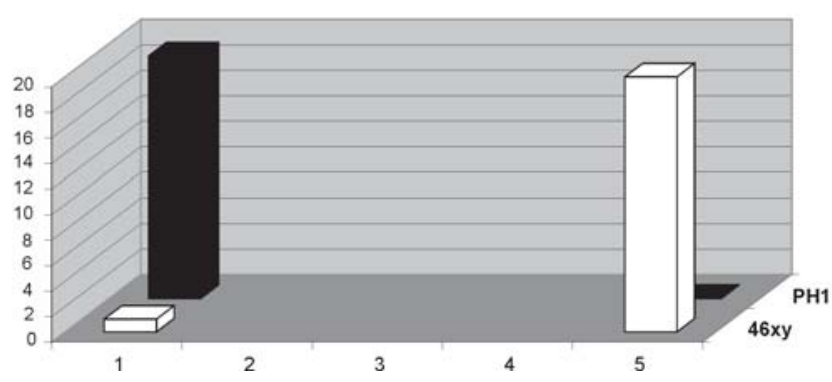

Gráfico 2. Evolução citogenética do caso 2

Caso 3

Paciente do sexo masculino, 18 anos, pardo, previamente hígido até outubro de 2003, quando apresentou um quadro de esplenomegalia, obtendo o diagnóstico de LMC $\mathrm{Ph} 1$ positivo em fase acelerada da doença. Iniciou o tratamento com hidroxiuréia, interferon alfa e arabinosídeo-c, apresentando apenas resposta parcial ao mesmo. Em abril de 2004 foi, então, realizado TMO alogênico com um score de três fatores de risco para o procedimento pelo EBMTR. O regime de condicionamento utilizado foi com bussulfano (16 mg/kg) e ciclofosfamida (120 mg/kg) e, em 05/04/2004, recebeu 3,06 x $10^{8}$ células nucleadas totais por kg de sua irmã HLA totalmente compatível. Teve como profilaxia da DECH esquema com metotrexato $15 \mathrm{mg} / \mathrm{m}^{2}$ no dia +1 e $10 \mathrm{mg} / \mathrm{m}^{2}$ nos dias $+3,+6$ e +11 aplicado por via endovenosa associado ao uso de ciclosporina. Evoluiu sem intercorrências até que, em novembro de 2004, apresentou alteração laboratorial compatível com recidiva da LMC em fase acelerada. Realizou o tratamento inicial com hidroxiuréia até preparo para infusão de DLI, porém, devido à dificuldade para o controle hematológico, foi modificado esquema para MI. Infelizmente, o paciente não obteve controle hematológico da doença mesmo com 800 mg/dia da droga e, diante dessa situação, foi programado um segundo TMO alogênico (Gráfico 3).

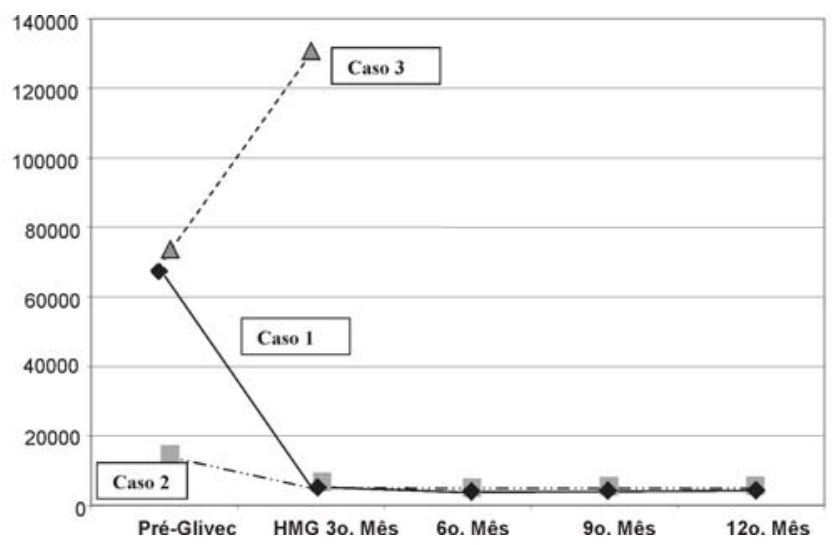

Gráfico 3. Evolução da leucometria dos casos relatados

\section{Discussão}

A LMC se caracteriza por uma doença mieloproliferativa crônica de difícil controle, entretanto, o TMO ainda é considerado, por muitos autores, como único tratamento capaz de promover a cura desta doença. ${ }^{9-14}$ Apesar dos altos índices de cura, as taxas de recidiva após o TMO são significativas, ${ }^{15}$ fazendo-se necessária uma terapêutica eficaz no manejo dessas situações.

A DLI tem sido, até então, a terapia de escolha para a recidiva de LMC pós-TMO. ${ }^{13-17}$ Apesar de poder induzir uma remissão considerável e duradoura, seu uso tem sido limitado pela associação com a doença do enxerto contra hospedeiro (DECH) e mielossupressão. ${ }^{13,14}$ Uma grande proporção de pacientes estudados que receberam terapia prévia com DLI e falharam no controle da recidiva, obteve resposta com o mesilato de imatinibe. Este fato tem levado alguns autores a estabelecer comparações entre essas duas terapias, ${ }^{13,14,15,21}$ sugerindo uma resposta comparável entre as duas modalidades de tratamento, porém com uma menor proporção de efeitos adversos associados ao uso o mesilato de imatinibe, ${ }^{15,21,22}$ o que fala a favor de seu uso como tratamento de primeira linha para recidiva de LMC após TMO.

Devido ao caráter recente do tema, ainda não foi possível avaliar a persistência dos resultados por um período maior de tempo. Estudos com maior período de segmento seriam necessários para elucidar esta dúvida.

Apesar de resultados favoráveis, estudos controlados, com maior poder de evidência, que comparem o mesilato de imatinibe com a DLI, seriam de fundamental importância para definição de conduta no manejo terapêutico desses pacientes. Os casos acima relatados podem ser levados em consideração para que se possam estabelecer novos critérios quan- 
to à terapia de escolha no tratamento da LMC recidivada após o TMO.

\begin{abstract}
Imatinib mesylate (MI), a selective tyrosine kinase inhibitor involved in the pathogenesis of chronic myelogenous leukemia (CML), has become the first-line treatment for this disease. Donor lymphocyte infusion (DLI) has been considered as the standard treatment for relapse after allogeneic bone marrow transplantation (BMT), even though it is frequently associated with graft versus host disease and myelosuppression. Because of the satisfactory results and tolerance of the treatment of CML, the authors used MI as an alternative therapy for DLI in patients that relapsed after BMT. They obtained cytogenetic remission in two cases, with, in one case, proven conversion to the donor chimera. The third case evolved with progression of the disease and a second BMT was required. Since this is a new alternative, this descriptive study suggests it should be considered as an alternative therapy for relapse after BMT. (Rev. bras. hematol. hemoter. 2006;28(2):157-160.
\end{abstract}

Key words: Chronic myeloid leukemia; treatment; relapse; bone marrow transplantation; imatinib mesylate.

\section{Referências Bibliográficas}

1. Fader $\mathrm{S}$ et al. The biology of chronic myeloid leukemia. N Eng J Med 1999;341(3):164-72.

2. Keating MJ. As leucemias crônicas. In: Goldman L, Bennett JC. Tratado de Medicina Interna, $21^{\circ}$ ed. Rio de Janeiro: Guanabara Koogan 2001;1.049-59.

3. Sawyers CL. Chronic myeloid leukemia. N Eng J Med 1999;340 (17):1.330-40.

4. Wetzler M, Byrd JC, Bloomfield CD. Leucemia mielóide aguda e crônica. In: Braunwald E, Fauci AS, Kasper DL, et al. Medicina Interna, $15^{\circ}$ ed. Rio de Janeiro: McGraw-Hill Companies 2002;749-59.

5. Savage DG, Avtman KH. Imatinib mesylate - a new oral target therapy. N Eng J Med 2002;346(9):683-93.

6. Pegs K, Mackinnon S. Imatinib mesylate - the new gold standard for treatment of chronic myeloid leukemia. N Eng J Med 2003; 348:1.048-50.

7. Kantarjian H, Sawyers C, Hochhaus A, et al. Hematologic and cytogenetic responses to imatinib mesylate in chronic myelogenous leukemia. N Eng J Med 2002;346(9):645-52.

8. O'Brien SG, et al. Imatinib compared with interferon and low-dose cytarabine for newly diagnosed chronic-phase chronic myeloid leukemia. N Eng J Med 2003;348:994-1004.

9. Souza CA, Pagnano K. Challenges in current chronic myeloid leukemia therapy of imatinib era. Rev Bras Hematol Hemoter 2004;26(4):282-4.

10. Goldman JM, Melo JV. Chronic myeloid leukemia - advances in biology and new approaches to treatment. N Eng J Med 2004; 349:1.451-64.

11. Kim Y, et al. Early prediction of molecular remission by monitoring BCR-ABL transcript levels in patients achieving a complete cytogenetic response after imatinib therapy for posttransplantation chronic myelogenous leukemia relapse. Biol of Bone Marrow Transplant 2004;10:718-25.

12. Kim Y, et al. Cytogenetic clonal evolution alone in CML relapse post-transplantation does not adversely affect response to imatinib mesylate treatment. Bone Marrow Reansplant 2004;33:237-42.
13. Olavarria E, et al. Imatinib mesylate (STI571) in the treatment of relapse chronic myeloid leukemia after allogeneic stem cell transplantation. Blood 2002;99(10):3.861-2.

14. Olavarria E, et al. Response to imatinib in patients who relapse after allogeneic stem cell transplantation for chronic myeloid leukemia. Leukemia 2003;17:1.707-12.

15. Kantarjian HM, O’Brien S, Cortes JE et al. Imatinib mesylate therapy for relapse after allogeneic stem cell transplantation for chronic myelogenous leukemia. Blood 2002;100(5):1.590-5.

16. Ullmann AJ, et al. Current results on the use of imatinib mesylate in patients with relapse Philadelphia chromosome positive leukemia after allogeneic or synergic hematopoietic stem cell transplantation. Keio J Med 2003;53(3):182-8.

17. Castro FA, et al. Immunological effects of donor lymphocyte infusion in patients with chronic myelogenous leukemia relapsing after bone marrow transplantation. Braz J Med Biol Res 2004;37 (2):201-6.

18. Au WY, et al. Tyrosine kinase inhibitor STI571 in the treatment of Philadelphia chromosome-positive leukemia failing myeloablative stem cell transplantation. Bone Marrow Transplant 2002; 30:453-7.

19. Wassmann B, et al. stable molecular remission induced by imatinib mesylate (STI571) in a patient with CML lymphoid blast crisis relapsing after allogeneic stem cell transplantation. Bone Marrow Transplant 2003;31:611-4.

20. Wassmann B, et al. Hematologic and cytogenetic remission by STI571 (Glivec) in a patient relapsing with accelerated CML after allogeneic stem cell transplantation. Bone Marrow Transplant 2001;28:721-4.

21. Deangelo DJ, et al. Extended follow-up of patients treated with imatinib mesylate (GLeevec) for chronic myelogenous leukemia relapse after allogeneic transplantation: durable cytogenic remission and conversion to complete donor chimerism without graft-versus-host disease. Clin Cancer Res 2004;10:5.065-71.

22. Kim, Y, et al. Cytogenetic clonal evolution alone in CML relapse post-transplantation does not adversely affect response to imatinib mesylate treatment. Bone Marrow Transplant 2004;33:237-42.

Avaliação: Editor e dois revisores externos.

Conflito de interesse: não declarado

Recebido: 05/02/2006

Aceito após modificações: 06/03/2006 